\title{
Multi-Viewpoint Smartphone AR-Based Learning System for Astronomical Observation
}

\author{
Ke Tian, Mamoru Endo, Mayu Urata, Katsuhiro Mouri, and Takami Yasuda
}

\begin{abstract}
Observational-based learning (OBL) can improve learners' understanding of astronomical phenomena, such as the lunar phases or solar diurnal motion. This paper introduces the multi-viewpoint smartphone AR-based (M-VSAR) learning system for constructing an OBL environment for astronomical observation. We apply this concept to two learning systems: a solar observation system and a lunar observation system. We illustrate how an M-VSAR system can enhance a learner's interest in and learning of astronomical phenomena.
\end{abstract}

Index Terms-Smartphone augmented reality, astronomy observation, lunar phases, solar motion, multi-viewpoint.

\section{INTRODUCTION}

Astronomers and planetarium curators believe that an education in astronomy requires outdoor observation [1], [2]. Observation-based learning (OBL) can improve a learner's understanding of astronomical phenomena, such as the lunar phases or solar diurnal motion. However, naked eye observation is often restricted by time, place, or weather. For example, learners typically observe celestial bodies in the sky at night, and such observation is subject to the interference of light pollution, blocking of highlands, climates, and seasons. Therefore, it is optimal to construct a real-world-oriented learning environment for learners to effectively grasp astronomical concepts.

This paper introduces the multi-viewpoint smartphone augmented-reality-based (M-VSAR) learning system for constructing an OBL environment for astronomy education. Then, we introduce two learning systems that apply M-VSAR: a solar observation system and a lunar observation system. The purpose of this paper is to illustrate how an M-VSAR system can enhance learner interest in and learning of astronomical phenomenon.

\section{RELATED WORK}

Augmented reality (AR) applies to "technologies that combine the real and the virtual in any location-specific way,

Manuscript received December 9, 2013; revised February 7, 2014. This work was supported by the Grants-in-Aid for Scientific Research Japan (No. 25280131).

K. Tian and T. Yasuda are with the Graduate School of Information Science, Nagoya University, Nagoya, 464-8601 Japan (e-mail: tianke0711@gmail.com, yasuda@is.nagoya-u.ac.jp).

M. Endo is with the School of Engineer, Chukyo University, Toyota, 470-0393 Japan (e-mail: endo@om.sist.chukyo-u.ac.jp).

M. Urata is with the Graduate School of International Development, Nagoya University, Nagoya, 464-8601 Japan (e-mail: mayu @ nagoya-u.jp).

K. Mouri is with the Astronomy Section, Nagoya City Science Museum, Nagoya, 460-0008 Japan (e-mail: mouri@nagoya-p.jp). where both real and virtual information play significant roles" [3].

AR's wide variety of uses includes demonstrating spatial concepts, temporal concepts, and contextual relationships between real and virtual objects. The main advantages of using virtual objects are that they can be animated, respond to user actions, and are not constrained by the costs, time, and practical or physical limitations of real objects. These factors make AR a powerful tool in astronomy education [4], [5].

Present-day smartphones and tablets integrate fast processors with graphics hardware, large touchscreens, and onboard sensors (cameras, GPS, compasses, and accelerometers) to create gadgets that are ideal for both indoor and outdoor AR experiences. Popular AR astronomy applications, such as Google Sky Map and Star Walk use a smartphone's GPS and sensors to locate the stars. However, these applications are not designed to be educational and will not help students learn about planet and star movement. Consider, for example, the lunar phases. There are no functions that show one-month moon orbit, shape, and age in the AR view. Further, these applications do not provide a function that explains the mechanism of lunar phases as it relates to their everyday observation.

The M-VSAR learning system assists learners in observing and studying the stars and planets, and achieves the educational goals of planetary and star movement in a real-world environment.

\section{M-VSAR LEARNING SYSTEM}

\section{A. Concept of a Learning System}

Setozakai [6] proposed helping learners understand astronomy topics from multiple viewpoints - namely, the geocentric model and the heliocentric model viewpoint. According to Setozakai's theory, it is important to observe planet and star movement from the viewpoint of the earth. At the same time, when studying the mechanisms of planet or star movement, learners should understand the relationships among the sun, earth, and other planets from the viewpoint of the sun as the center of the universe.

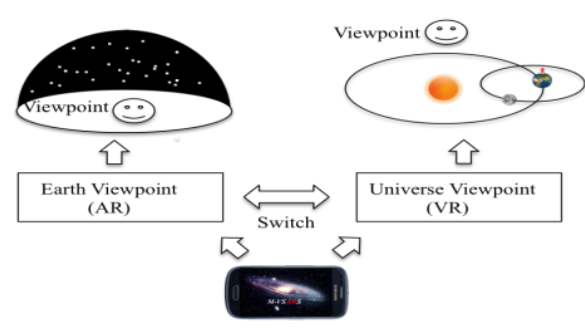

Fig. 1. Outline of the M-VSAR system. 
Therefore, we used smartphone AR and Virtual Reality (VR) contents to develop the M-VSAR learning system for planetary motion education. Fig. 1 gives an outline of M-VSAR system. Learners can switch between the two viewpoints to observe celestial bodies and achieve the learning goal of celestial body motion.

\section{B. Functional Design}

To achieve the learning goal of planetary motion observation, we developed system functions based on learning indicators in the astronomy unit of Japan's junior high school science course. Each function matches a learning goal of an astronomy observation task. For example, the learning goals of lunar phases include observing the moon every day to witness its periodic waxing and waning. To this end, in the M-VSAR learning system's lunar cycle simulation, learners can observe image shape, moon age, azimuth, and elevation angle in the AR view using the "SetDay" function.

\section{Astronomy Simulation Model}

Our research group focuses on the simulation of astronomical phenomenon. In this research, we use the astronomy simulation model to develop the M-VSAR learning system, shown in Fig. 2.

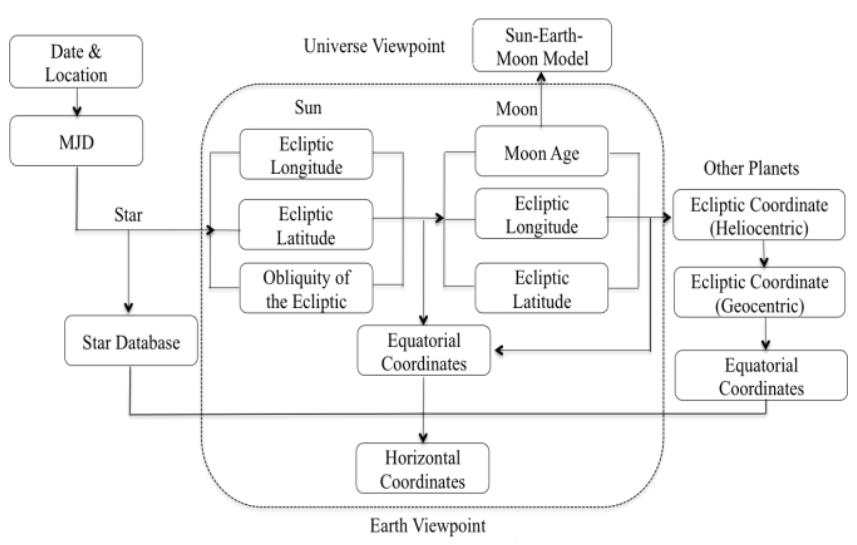

Fig. 2. Astronomy simulation model.

\section{AR View}

The M-VSAR learning system shows virtual planetary images, such as the moon overlaid on a live video camera background, making the virtual celestial bodies image appear to be in the real world. Visualizing celestial bodies correctly in the AR view requires obtaining real-time data from a smartphone's built-in sensors. This data is then combined with the astronomy simulation model (see Fig. 2) to calculate the screen coordinates of the visual celestial bodies in AR view.

Fig. 3 shows the procedure for calculating the position of celestial bodies. First, it is necessary to obtain the date and time from the smartphone's calendar and calculate the Modified Julian Day (MJD). The longitude and latitude data is then obtained using the GPS sensor, which is responsible for tracking the smartphone's geographical position. By combining these data with the horizontal coordinates algorithm (ALG-2), the system can calculate the moon age, azimuth, and elevation of the moon and sun et al., in real time. The azimuth and elevation data is the horizontal coordinate data of the celestial bodies. This data is then used to derive the data of the screen coordinate of the planet and to register the planet in the $\mathrm{AR}$ view, corresponding to the actual celestial bodies in the sky. The accelerometer and compass sensors are used to measure the viewing direction and elevation angle of the smartphone device with which the user is observing the celestial bodies. Based on this tracking information, we use the screen projection algorithm (ALG-3) to convert the horizontal coordinates to screen coordinates to locate the celestial bodies in the AR view.

We used the M-VARML learning system for solar observation to explain the AR view interface. Fig. 4 shows the complete AR view interface of M-VARML.

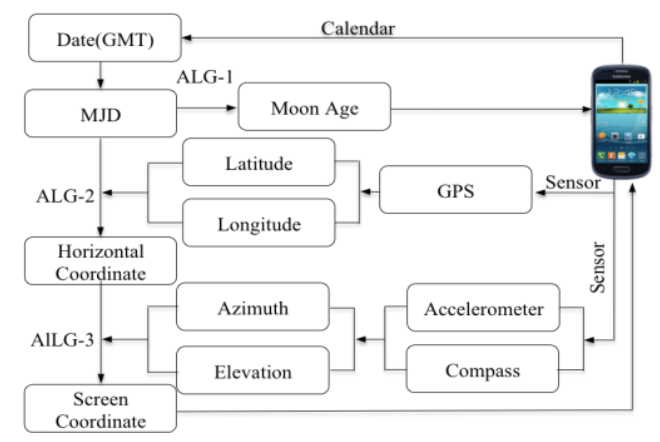

Fig. 3. Calculating the position of planets in AR view.

The main visual components overlaid in the AR view include the data part, visual sun image, latitude line, longitude line, and horizon line. Text at the top right of the screen lists data on the orientation, elevation angle of the mobile device, azimuth, and elevation of the sun. In addition, learners can change the latitude and longitude, text size, color, and so on, in the settings interface.

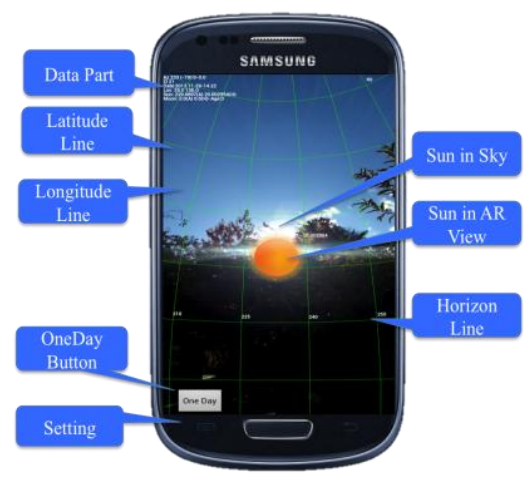

Fig. 4. AR view interface.

\section{E. M-VSAR for Lunar Observation}

Learning indicators in the student's science course unit entitled "Lunar Phase" include the following tasks:

1) Observe the moon, its azimuth, and elevation angle in the sky every day and witness it rising in the east and setting in the west.

2) Observe the moon every day to understand its periodic waxing and waning over 30 days.

3) Understand the relationship between moon age and shape of lunar phases.

4) Understand the positional relationship among the sun, earth, and moon to study the mechanism of lunar phases from the universe viewpoint. 


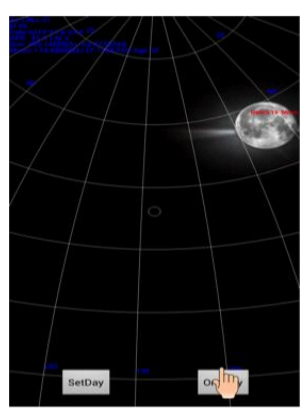

(a)
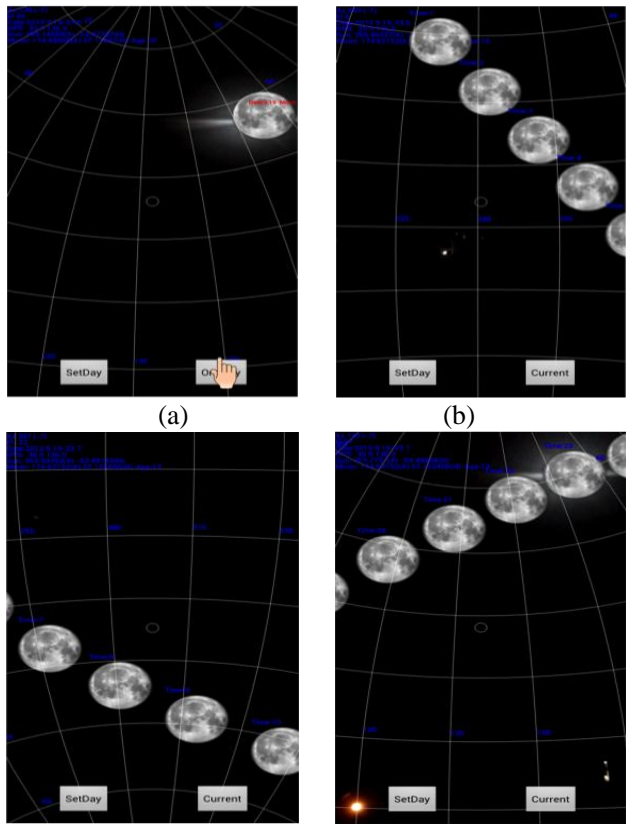

(c)

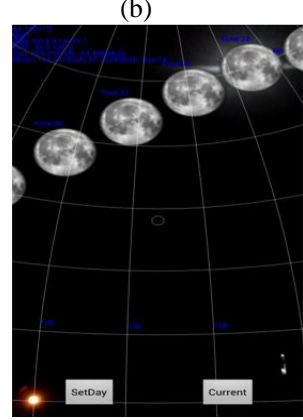

(d)

Fig. 5. Observe one day moon orbit in AR view.
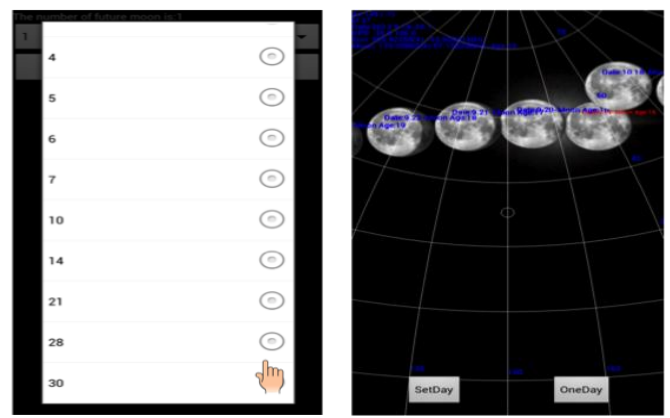

(a)

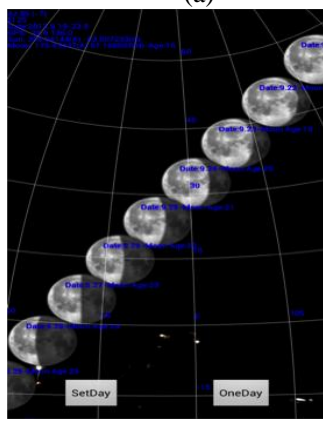

(c)

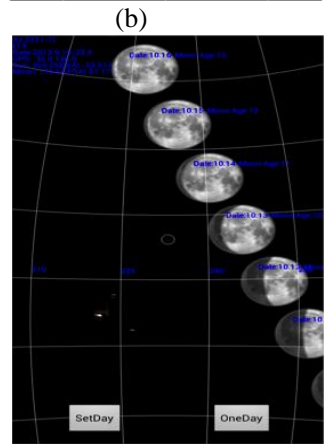

(d)

Fig. 6. Observe the waxing and waning of the moon in AR view.

The learner rotates the mobile device to observe the moon's orbit from 1:00 a.m. to 12:00 p.m. using the "OneDay" function shown in Fig. 5. This function helps learners achieve learning goal (2). In AR view, learners can observe the periodic waxing and waning of the moon, the orbit, azimuth, elevation angle, age, and shape of consecutive moons in the sky in a particular location simultaneously using the "SetDay" function, as shown in Fig. 6. This function helps learners achieve learning goals (2) and (3).

The best way to understand the phases of the moon is to examine an earth-moon-sun view. We developed the universe view to show the relationship of the sun-earth-moon orbit based on moon age. When users touch the current moon image in the AR view, they see an item named "mechanism." On selecting this item, the user steps into the universe view interface as showed in Fig. 7.
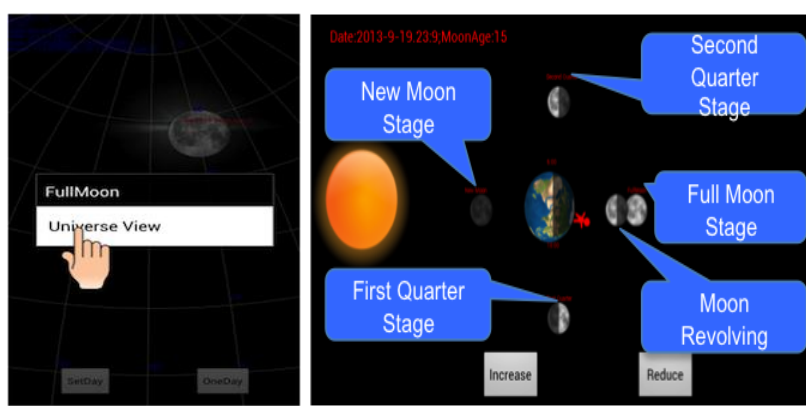

Fig. 7. Universe view interface (Lunar).

Learners can use the increase and reduce buttons to move the moon in the orbit. The universe view meets the stated specification of the learning environment in leaning goal (4).

\section{F. M-VSAR for Solar Observation}

Learning indicators in the student's science course unit entitled "Solar Movement" include the following learning goals:

1) Observe the sun every day to witness it rising in the east and setting in the west, the noon altitude of the sun, and daytime length.

2) Observe the solar diurnal motions orbit, noon altitude of the sun, and daytime length in the four seasons.

3) Observe the solar diurnal motions orbit, daytime length, and noon altitude of the sun in different locations - namely, from the user's current location, the equator, and the North Pole.

4) Understand the relationship between the sun and the earth in the universe view.

To achieve the solar observation learning goals, we developed several specific functions in the M-VSAR solar system. When users touch the "current" button, as shown in Fig. 8(a), the one-day sun orbit is displayed in the AR view, as shown in Fig. 8(b). This function helps learners achieve learning goal (1). Solar diurnal motion orbits in spring, summer, autumn, and winter are displayed in the AR view by the "ShowSeasonNoonAltitude" function, as Fig. 9 shows. Learners can observe the solar orbit, noon altitude of the sun, daytime length, and rising and setting of the sun in each of the four seasons. This function enables learners to achieve learning goal (2). Learners can observe the diurnal motion of the sun in different locations at the same time using the "ShowLocationNoonAltitude" function. The current location and the solar motion orbit at the North Pole and equator are displayed in AR view, as Fig. 10 shows. For example, in this view, learners can intuitively grasp that the solar diurnal motion orbit at the North Pole is parallel to the equatorial line (the yellow line in Fig. 10. This function enables learners to achieve learning goal (3).

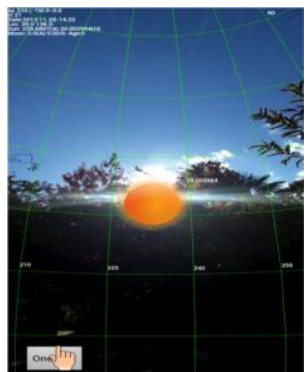

(a)

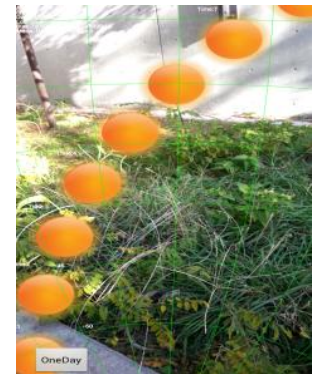

(b) 


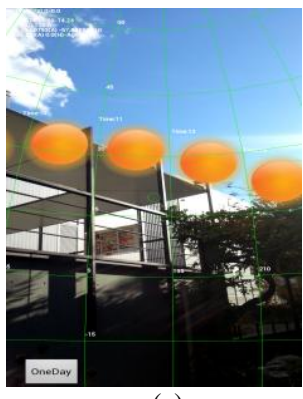

(c)

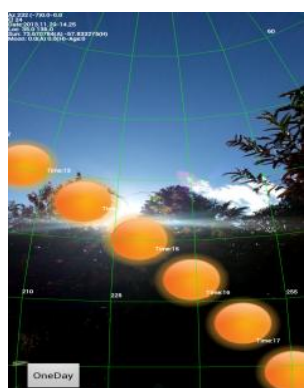

(d)

Fig. 8. One day solar orbit in AR view

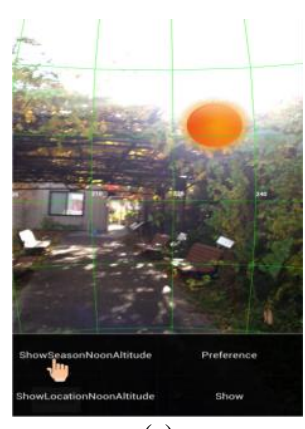

(a)

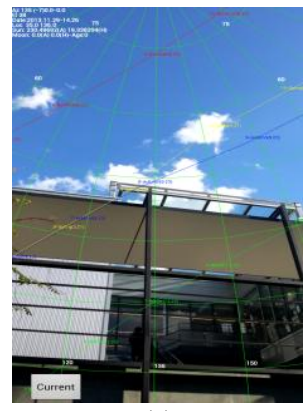

(c)

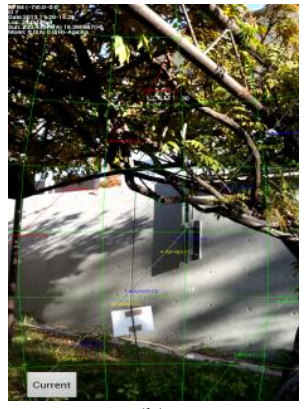

(b)

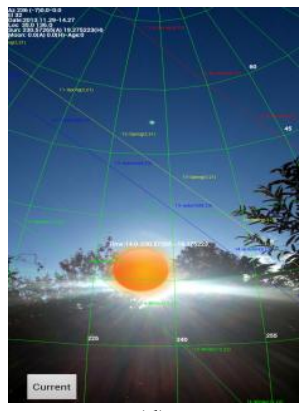

(d)
Fig. 9. Solar diurnal motion orbit in spring, summer, autumn, and winter.

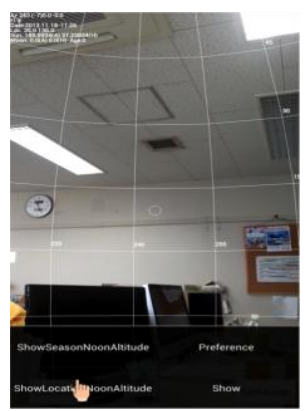

(a)

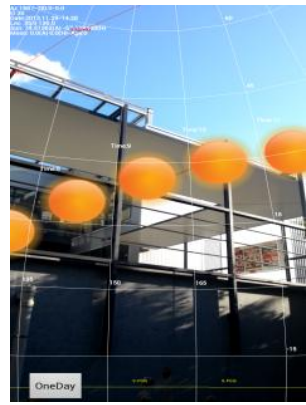

(c)

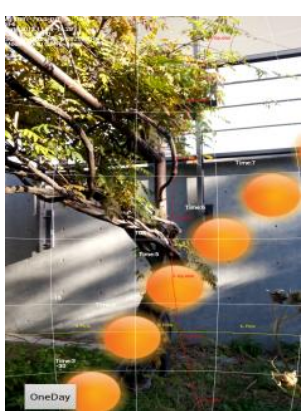

(b)

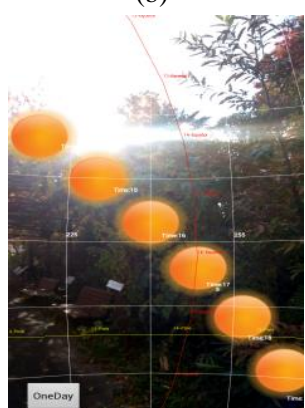

(d)
Fig. 10. Solar diurnal motion orbit of observer location, equator, and North Pole.

Previous work showed that using the 3D modeling could help students solve their misconceptions about astronomy concepts, such as the solar system [7]. Based on the sun-earth orbit astronomy model, we used the Android OpenGL to develop a real-time 3D sun-earth movement simulator to assist learners in understanding the earth's revolution and rotation in the universe view. When users touch the current sun image in the AR view, they see a "mechanism" item. On selecting this item, the user steps into the universe view interface, as shown in Fig. 11. The universe view satisfies learning goal (4). This improves learners' knowledge regarding the mechanism of solar diurnal motions.

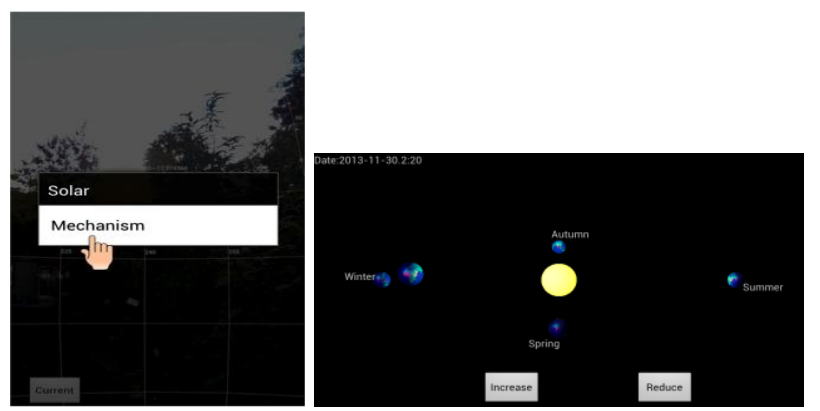

Fig. 11. Universe view interface (solar).

\section{SYSTEM IMPLEMENTATION}

We developed the M-VSAR learning system on the Google Android operating system and software development kit. The developing code toolset is Eclipse, ADT, and Android 2.1 SDK. The programming language is Java. The $3 \mathrm{D}$ models of the sun and earth were developed using the Android OpenGL API. The application is supported by a range of smartphones and tablet devices running on Android 2.1 or higher, and have the required sensors (i.e., camera, GPS, electronic compass, and accelerometer). Our M-VARML system was implemented on an Android smartphone. The mobile device was a Samsung Galaxy S3.

\section{SYSTEM TEST AND EXPERIMENT}

Two kinds of tests were conducted. We conducted one to confirm the system's accuracy and stability, whereas test subjects conducted a task-based experiment to verify the system's usability and usefulness. Fig. 12 shows the test example image.
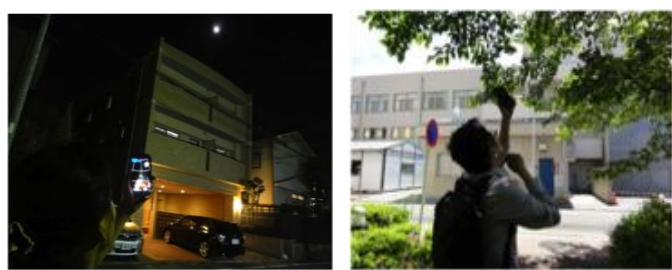

Fig. 12. Experimental scene.

Because the system's stability and accuracy is critical to learners, we tested for these two factors. First, we tested the two systems in various locations and at various times. Through these tests, we confirmed that the solar and lunar images matched well with the sun and moon in the sky. To ensure stability of the AR view, we developed a level of sensor sensitivity option function.

Furthermore, previous research [8] conducted by the authors of the present paper confirmed the usefulness, usability, and effectiveness of the M-VSAR learning system 
for lunar observation. We conducted task-based experiments to evaluate the above system factors through questionnaires. Results of the questionnaires indicated that most participants thought the system's functions were very effective. Participants agreed that the system could help them improve their knowledge of lunar phases and erase some of their misconceptions, thus confirming the system's usefulness. Moreover, the users agreed that the system was easy to operate. They were able to operate the system to complete the moon observation tasks, confirming the system's usability. Lastly, the participants appeared to be highly interested in using the system to observe lunar phases. Participants agreed that the system helped them enhance their motivation to observe lunar the in their daily life.

The above experimental results suggest that the M-VSAR system for astronomy observation can positively affect learning and motivation.

\section{CONCLUSION}

In this paper, we proposed the concept of M-VSAR learning system. We utilized smartphone AR and VR content to develop two systems, one for lunar, and one for solar motion observation.

We conducted experiments to test the M-VSAR system's accuracy, stability, usefulness, and usability, as well as user satisfaction with the system. The results suggest that the M-VSAR system for astronomical observation can positively affect learning and motivation.

For future work, we plan to improve the system's interface design so even learners with limited knowledge can use it. Our future challenges include the implementation of the M-VSAR learning system in middle school science classes.

\section{ACKNOWLEDGMENT}

Thanks to Mr. Tom Kabara of Mei Writing in Nagoya University for patiently guiding us in writing our paper.

\section{REFERENCES}

[1] M. Kondo, M. Mouri, and Y. Yausda, "A Multiplatform Content Management System for Curators to Provide Teaching Materials in Astronomy Education," Journal of Theoretical and Applied Information Technology, vol. 4, no. 9, pp. 874-881, 2008.

[2] M. Soga, M. Ohama, Y. Ehara, and M. Miwa, "Real-World Oriented Mobile Constellation Learning Environment Using Gaze Pointing," IEICE Transactions on Information and Systems, vol. 94, no. 4, pp. 763-771, 2011

[3] E. Klopfer and K. Squire, "Environmental detectives-The development of an augmented reality platform for environmental simulations," Educational Technology Research and Development, vol. 56, no. 2, pp. 203-228, 2008.

[4] B. E. Shelton and N. Hedley, "Using augmented reality for teaching earth-sun relationship to undergraduate geography students," in Proc.
First IEEE International Augmented Reality Toolkit Workshop, Darmstadt, Germany, 29 September 2002.

[5] A. K. Sin and H. B. Zaman, "Tangible interaction in learning astronomy through augmented reality book-based educational tool," in Proc. First International Visual Informatics Conference, IVIC 2009, Kuala Lumpur, Malaysia, 11-13 November 2009, pp. 302-313.

[6] N. Setozakai, Y. Morita, and T. Taketa, "Study on effective use scene of multi aspect type VR teaching materials in astronomical education," Institute of Electronics Information and Communication Engineers Technical Report, vol. 107, no. 391, pp. 51-56, 2007.

[7] M. Barnett, "Using virtual reality computer models to support student understanding of astronomical concepts," Journal of Computers in Mathematics and Science Teaching, vol. 24, no. 4, pp. 333-356, 2005.

[8] K. Tian, M. Endo, M. Urata, K. Mouri, and T. Yasuda, "Ubiquitous Augmented Reality Mobile Learning System for Supporting Moon Observation," in Proc. International Conference on Information and Social Science, September 2013, pp. 223-242.

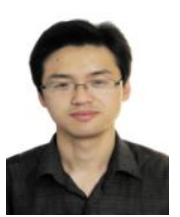

Ke Tian is a $\mathrm{PhD}$ candidate in the Graduate School of Information Science at Nagoya University, Japan. His research interests include smartphone AR, mobile human-computer interaction (HCI), and ubiquitous learning. Tian received a master's degree in engineering from Dalian University of Technology, China in 2009.

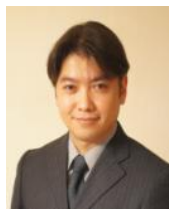

Mamoru Endo is an associate professor in the school of Engineering at Chukyo University, Aichi, Japan. He received his Ph.D. degree from Nagoya University. His research interests include network systems, computer graphics, virtual reality and their application in real society.

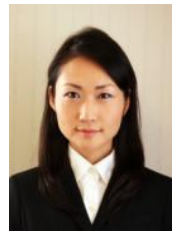

Mayu Urata is an assistant professor in the Graduate School of International Development at Nagoya University, Japan. She received the Master and Ph.D. degrees in information science from Nagoya University, in 2008 and 2011, respectively. Her research interests include social and educational applications of information and communication technologies.

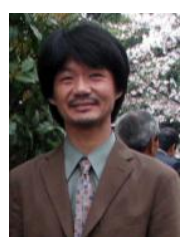

Katsuhiro Mouri is the chief curator of the Planetarium in Nagoya City Science Museum. His education includes: BS and MS in earth and planetary science, Nagoya University, Nagoya, Japan in 1986 and 1988. He received his Ph.D. in human informatics, Nagoya University in 2011.His business experience includes: System engineer of Computer system NTTDATA Corp. 1988-1990.

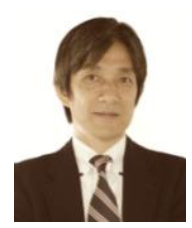

Takami Yasuda is a professor in the Graduate School of Information Science and School of Informatics and Sciences at Nagoya University. Yasuda received his Ph.D in information engineering from Nagoya University in 1989. His research interests include social and educational applications of information and communication technologies. He is the councilor of Nagoya University and the vice dean of the Graduate School of Information Science, Nagoya University. 